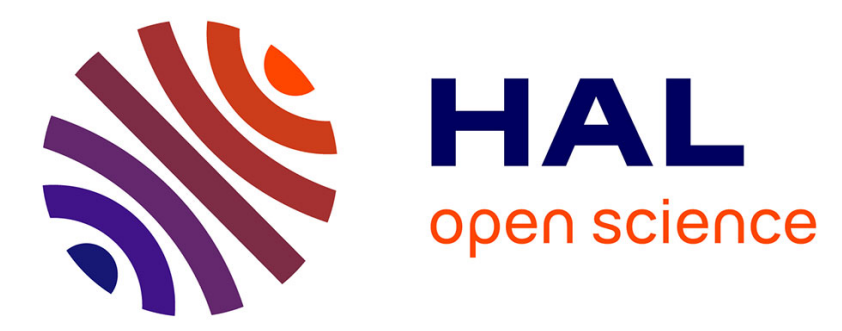

\title{
A time-incremental Eshelby-based homogenization scheme for viscoelastic heterogeneous materials
}

\author{
Stéphane Berbenni, Hafid Sabar
}

\section{To cite this version:}

Stéphane Berbenni, Hafid Sabar. A time-incremental Eshelby-based homogenization scheme for viscoelastic heterogeneous materials. Micromechanics and Nanomechanics of Composite Solids, 2017, 978-3-319-52793-2. hal-02360507

\section{HAL Id: hal-02360507 https://hal.science/hal-02360507}

Submitted on 12 Nov 2019

HAL is a multi-disciplinary open access archive for the deposit and dissemination of scientific research documents, whether they are published or not. The documents may come from teaching and research institutions in France or abroad, or from public or private research centers.
L'archive ouverte pluridisciplinaire HAL, est destinée au dépôt et à la diffusion de documents scientifiques de niveau recherche, publiés ou non, émanant des établissements d'enseignement et de recherche français ou étrangers, des laboratoires publics ou privés. 


\title{
A time-incremental Eshelby-based homogenization scheme for viscoelastic heterogeneous materials
}

\author{
Stéphane Berbenni and Hafid Sabar
}

\begin{abstract}
A time-incremental Eshelby-based homogenization scheme for Maxwellian heterogeneous materials is proposed and discussed. This is based on the exact solution of the heterogeneous Eshelby ellipsoidal inclusion problem obtained in the time domain. In contrast with hereditary methods, the effective behavior as well as the evolution laws of the averaged stresses per phase are solved incrementally in the time domain without need to inverse Laplace or Laplace-Carson transforms. This is made through a time-differential equation to exactly solve a volume term in the integral equation that was generally approximated in previous internal variable methods. The present formulation works for any arbitrary anisotropic ellipsoidal Maxwellian inclusion embedded in an isotropic Maxwellian matrix without any other restrictive assumptions. In order to show the interest of the present approach, a Mori-Tanaka homogenization scheme is applied to two-phase composites using the developed strain rate concentration equations. The results are reported and discussed in comparisons with other existing methods, including hereditary approaches and more recent internal variable approaches, in order to show the efficiency of the present time-incremental homogenization scheme.
\end{abstract}

\section{Introduction}

Materials like composites, metals or polymers exhibit time-dependent behaviors which, from the homogenization point of view may require the use of linear viscoelastic properties [7, 22]. Generally, the coupling between elastic and inelastic deformations renders micromechanical homogenization schemes particularly complex even in the case of linear viscoelastic heterogeneous materials. For instance, it was highlighted that the overall behavior of aggregates with Maxwellian constituents is no longer Maxwellian [44, 40, 24]. This is due to the differential nature of constitutive equations which involves different orders of time derivation mechanical fields. In past decades, several approaches were proposed to solve linear viscoelastic problems.

Riemann-Stieltjes integral-based "hereditary" homogenization approaches using Laplace-Carson transforms and the correspondence principle (see e.g. [26]) were first developed in the case of non ageing linear viscoelastic materials. The inclusion problem was first solved [13, 22], and, further introduced in different homogenization schemes: Mori-Tanaka [47, 24, 25, 6, 37], Hashin-Shtrickman [9] or self-consistent $[22,50,45,40,41,29,5]$ estimates, among others. The overall properties of composites in the time domain are then obtained by the Laplace or Laplace-Carson inversion either analytically in simple cases $[13,40,47,24]$, or, numerically in major cases $[22,23]$ which demands large CPU time and memory.

A more efficient homogenization strategy is based on "incremental internal variables approaches" whereby the stress history is recorded through the inelastic strains of the individual constituents. The hereditary approaches give the solution in the transformed (Laplace or Laplace-Carson) domain whereas the

Stéphane Berbenni

Laboratoire d'Etude des Microstructures et de Mécanique des Matériaux, UMR CNRS 7239, Université de Lorraine, 57045 Metz, France e-mail: stephane.berbenni@univ-lorraine.fr

Hafid Sabar

Laboratoire de Mécanique Biomécanique Polymères Structures, Ecole Nationale Ingénieurs de Metz, 57078 Metz, France e-mail: sabar@enim.fr 
internal variables formulations directly provide the solution in the time domain. Time is implicitly present in these formulations through the inelastic strains. Thus, these fields are defined as "internal variables". One of the main advantage of developing such approaches in addition to CPU time and memory, lies in examining complex loading paths in time domains and aging behaviors.

First internal variables self-consistent and Mori-Tanaka approximations for heterogeneous linear and non linear viscoelastic materials were developed in $[48,14,33,36,42,32]$. The first developments were carried out by [48] in the eighties who adapted [16]'s elastoplastic self-consistent model to the case of elasto-viscoplasticity. However, similarly to the original proposition of [16], internal stresses are overestimated with this method. In the nineties, an "additive" interaction law for the Eshelby inclusion problem was derived for Maxwellian local viscoelastic behavior by Molinari et al. [14, 33, 32]. This interaction law was written in terms of strain rates and was postulated as the superposition of elastic and inelastic interaction laws. It was applied to the non linear elasto-viscoplastic Eshelby inclusion problem using a "tangent" formulation with some applications to the Mori-Tanaka and self-consistent estimates in [30]. The "translated fields" method was developed for linear and non-linear viscoelasticity in [36] and in [42] starting from the integral equation of the heterogeneous elasto-viscoplastic problem. Good predictions were found in [36] in comparison with the exact "1-site" self-consistent solution of [41]. The "translated fields" approach of [36] was recentlty extended to treat linear viscoelastic composites with the Mori-Tanaka scheme in [31], where it was compared to the "additive" interaction law and Fast Fourier Transform (FFT) calculations. A very rich but complex interaction law was obtained with the "translated fields" method in [31]. The extensions of the "translated fields" method to non linear viscoelastic composites and polycrystals with an "affine" formulation were recently reported by [2] and by [27], respectively. The results of this extended "translated fields" method were successfully compared to the ones obtained by the "affine" hereditary approach developed by [29] for the same non linear polycrystal [27]. Another approach based on a "sequential" linearization technique was recently developed by [15]. This technique is based on the sequential resolution of purely elastic and purely viscoplastic interaction laws. Different "variants" of the technique were proposed for sequential linearization and the aforementioned "additive" interaction law was retrieved as a specific "variant". Lastly, a self-consistent internal variables approach for two-phase linear isotropic viscoelastic composites was also proposed by [8] and successfully compared to the exact results of [13] and [41] for two-phase composites. Variational approaches were developed by $[19,20]$ and by [4]. An incremental internal variables approach was first developed in linear viscoelasticity by [19] and extended to non linear viscoelasticity in [20]. These authors used two potentials (free energy density and dissipation potential) with an implicit time discretization scheme based on a variational method. Their theory was applied to both Maxwell and Kelvin-Voigt rheological models. The FFT technique was also reported in $[19,20]$ as "exact" reference solutions to assess the variational approach. Recently, another variational approach was developed in [4] for non linear two-phase elasto-viscoplastic composites using a "secant" linearization scheme and a Mori-Tanaka approximation. A method inspired from the collocation method was first developed by [39]. Interestingly, this approach is based on an equivalence between the collocation method and an internal variables formulation to obtain the exact effective behavior for a two-phase microstructure in the cases of Voigt, Reuss and Mori-Tanaka estimates. Exact analytical results were provided in [39] in the case of two-phase elastically homogeneous Maxwellian linear viscoelastic composites. These solutions will be used in the sequel to assess the present internal variables formulation with a Mori-Tanaka estimate. The extension of the [39]'s method to the case of linear viscoelastic polycrystals with a self-consistent procedure was recently reported in [28]. It is noteworthy that the [39]'s homogenization scheme was also applied to ageing linear viscoelasticity. A different homogenization scheme for aging linear viscoelastic matrix-inclusion composite materials using the Volterra operator and working in the time domain was developed by [43] for isotropic phases and spherical inclusions. This scheme was recently extended by [21] to ellipsoidal inclusions and assuming isotropic aging viscoelastic matrices with time-independent Poisson ratio.

The objective of this chapter is to present and discuss a recent internal variables approach first introduced by [3] based on a an incremental Eshelby-based homogenization scheme for viscoelastic heterogeneous materials operating in the time domain. Section 2 introduces the constitutive equations and the exact time-incremental internal variables formulation in the case of micro-heterogeneous linear viscoelastic Maxwellian behavior. The integral equation can be simplified by considering isotropic viscoelastic properties for the reference medium without other more restrictive assumptions. Then, the incremental internal variables approach is set up through a time differential equation related to the "volume" term in the integral equation. In section 3, the viscoelastic Eshelby inclusion problem is exactly solved through incremental strain rate concentration laws and through incremental interaction laws that does not contain any approxi- 
mation. Based on the strain rate concentration equations of the Eshelby problem, a time-incremental internal variables homogenization Mori-Tanaka scheme is derived in Section 4 for linear viscoelastic two-phase composites and an isotropic distribution of phases. Then, this homogenization scheme is applied to different cases, including comparisons with other Mori-Tanaka homogenization schemes for two-phase Maxwellian composites: (i) the exact analytical derivations from [24] using a hereditary approach and Laplace transform inversion, (ii) the [39]'s exact formulation for compressible viscoelastic phases with homogeneous elasticity, and (iii) the approximate models based on the "translated fields" method [36, 2, 27] or based on the "additive" interaction law [14, 33, 32, 30].

\section{Time-incremental formulation}

\subsection{Constitutive equations}

A heterogeneous linear viscoelastic medium $V$ with boundary $\partial V$ is considered with Maxwellian constituents characterized by linear elastic moduli $\underset{\approx}{c}$ (elastic compliances $\underset{\approx}{s}=\underset{\approx}{c}$ ) and linear inelastic moduli $\underset{\approx}{b}$ (inelastic compliances $\underset{\approx}{m}=\underset{\approx}{b^{-1}}$ ). For linear viscoelasticity, $\underset{\approx}{m}$ and $\underset{\approx}{b}$ are constant (i.e. time independent). Here, non-linear viscoelasticity, where $\underset{\approx}{\approx}$ and $\underset{\approx}{\approx}$ are time and stress-dependent, is disregarded. The problem is treated within the framework of quasi-static equilibrium with infinitesimal strains and no volume forces. Thus, for a given applied homogeneous strain rate $\underset{\sim}{\dot{\varepsilon}^{0}}$, field equations of the linear viscoelastic (Maxwellian) problem are given by:

$$
\begin{aligned}
& \underset{\sim}{\sigma}=\underset{\approx}{b}:(\underset{\sim}{\dot{\varepsilon}}-\underset{\sim}{s}: \dot{\sim}) \text { or } \underset{\sim}{\dot{\sigma}}=\underset{\approx}{c}:(\underset{\sim}{\dot{\varepsilon}}-\underset{\approx}{m}: \underset{\sim}{\sigma}) \\
& \underset{\sim}{\operatorname{div}} \underset{\sim}{\dot{\sigma}}=0 \\
& \underset{\sim}{\operatorname{div} \sigma} \underset{\sim}{\sigma}=0 \\
& \underset{\sim}{\dot{\varepsilon}}=\nabla^{s} \underline{\dot{u}} \\
& \underline{\dot{u}}^{d}=\dot{\sim}^{\dot{\varepsilon}^{0}} \cdot \underline{x} \text { on } \partial V
\end{aligned}
$$

where $\underset{\sim}{\dot{\varepsilon}}$ is the total strain rate with linearized kinematics, $\underset{\sim}{\sigma}$ is the Cauchy stress, $\underset{\sim}{\dot{\varepsilon}^{e}}=\underset{\sim}{s}: \underset{\sim}{\dot{\sigma}}\left(\operatorname{resp} . \underset{\sim}{\dot{\varepsilon}^{v}}=\underset{\approx}{m}: \underset{\sim}{\sigma}\right)$ is the elastic (resp. inelastic) strain rate. In Eq. 1, $\underline{u}$ is the unknown displacement field, $\nabla^{s} \underset{\sim}{\sim}$ denotes the symmetrized gradient operator, $\underline{\text { div }}$ denotes the divergence operator and the symbols “.”, “:” denote simple and contracted products, respectively.

The heterogeneous elastic moduli $\underset{\approx}{c}($ resp. compliances $\underset{\approx}{s})$ and the heterogeneous viscous moduli $\underset{\approx}{b}$ (resp. compliances $\underset{\approx}{m}$ ) can be written in terms of spatial fluctuations $\underset{\approx}{\delta c}($ resp. $\underset{\approx}{\delta} s)$ and $\underset{\approx}{\delta b}($ resp. $\underset{\approx}{\delta}$ ) with respect to the infinite homogeneous reference medium $\underset{\approx}{c^{0}}\left(\right.$ resp. $\left.\underset{\approx}{s^{0}}\right)$ as follows:

$$
\underset{\approx}{c}=\underset{\approx}{c^{0}}+\underset{\approx}{\delta} ; \underset{\approx}{s}=\underset{\approx}{s^{0}}+\underset{\approx}{\delta s} ; \underset{\approx}{b}=\underset{\approx}{b^{0}}+\underset{\approx}{\delta b} ; \underset{\approx}{m}=\underset{\approx}{m^{0}}+\underset{\approx}{\delta m}
$$

where $\underset{\approx}{c}, \underset{\approx}{s^{0}}, \underset{\approx}{b^{0}}$, and, $\underset{\approx}{m^{0}}$ denotes the elastic moduli, elastic compliances, viscous moduli, viscous compliances of the homogeneous reference medium. Using the classic Green's function technique and previous constitutive equations yields:

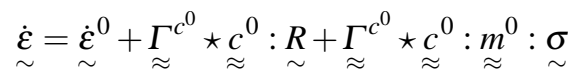

or:

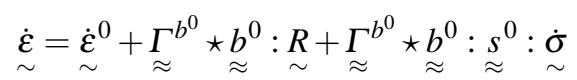

where $\star$ denotes spatial convolution and $R$ contains the fluctuations of both elastic and inelastic compliances and is given by: 


$$
\underset{\sim}{R} \underset{\approx}{\delta}: \underset{\sim}{\dot{\sigma}}+\underset{\approx}{\delta} m: \underset{\sim}{\sigma}
$$

The fourth-order tensor $\underset{\approx}{\Gamma^{0}}\left(\right.$ resp. $\left.\underset{\approx}{\Gamma^{b^{0}}}\right)$ is the modified Green tensor associated with $\underset{\approx}{c^{0}}\left(\operatorname{resp} . \underset{\approx}{b^{0}}\right)$ as defined in [17]:

$$
\Gamma_{i j k l}^{*}\left(\underline{x}-\underline{x}^{\prime}\right)=-\frac{1}{2}\left(G_{i k, j l}^{*}\left(\underline{x}-\underline{x}^{\prime}\right)+G_{j k, i l}^{*}\left(\underline{x}-\underline{x}^{\prime}\right)\right)
$$

where $\underset{\sim}{G^{*}}$ is the Green tensor (see [35]) associated with $*=c^{0}$ and $*=b^{0}$, and, $\underline{x}$ and $\underline{x}^{\prime}$ are position vectors.

\subsection{Time-incremental internal variables formulation}

For the rest of the paper, we assume the case of a homogeneous reference medium with isotropic elastic and viscous properties, where $k^{0}, k_{v}{ }^{0}$ are the elastic and inelastic bulk moduli of the reference medium, and, $\mu^{0}$, $\mu_{v}{ }^{0}$ are its elastic and inelastic shear moduli, such that:

$$
\begin{aligned}
& \underset{\approx}{c^{0}}: \underset{\approx}{m^{0}}: \underset{\sim}{\sigma}=\left(\frac{k^{0}}{k_{v}^{0}}-\frac{\mu^{0}}{\mu_{v}^{0}}\right) \underset{\sim}{\sigma} \underset{\sim}{\delta}+\frac{\mu^{0}}{\mu_{v}^{0}} \underset{\sim}{\sigma} \\
& \underset{\approx}{b^{0}}: \underset{\approx}{s}: \underset{\sim}{\dot{\sigma}}=\left(\frac{k_{v}^{0}}{k^{0}}-\frac{\mu_{v}^{0}}{\mu^{0}}\right) \underset{\sim}{\dot{\sigma}_{m}} \underset{\sim}{\delta}+\frac{\mu_{v}^{0}}{\mu^{0}} \underset{\sim}{\dot{\sigma}}
\end{aligned}
$$

where $\sigma_{m}$ is the spherical part of $\underset{\sim}{\sigma}$ (i.e. hydrostatic pressure $\sigma_{m}=\frac{1}{3} \sigma_{k k}$ ).

For any statically admissible stress field verifying Eq. 1, traction vector continuity at perfectly bonded interfaces and homogeneous boundary conditions, the modified Green operators associated with the homogeneous reference elastic and viscous media have the following property $[18,17]$ :

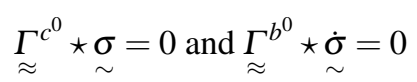

Using Eq. 7 and Eq. 8, Eq. 3 can be recast:

$$
\underset{\sim}{\dot{\varepsilon}}=\underset{\sim}{\dot{\varepsilon}^{0}}+\underset{\approx}{\Gamma^{c^{0}} \star} \underset{\approx}{c^{0}}: R+\beta \underset{\sim}{\sigma^{*}}
$$

with:

$$
\beta=\frac{3}{3 k^{0}+4 \mu^{0}}\left(\frac{k^{0}}{k_{v}^{0}}-\frac{\mu^{0}}{\mu_{v}^{0}}\right)
$$

and Eq. 4 simplifies to:

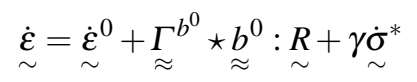

with:

$$
\gamma=\frac{3}{3{k_{v}}^{0}+4 \mu_{v}{ }^{0}}\left(\frac{k_{v}{ }^{0}}{k^{0}}-\frac{\mu_{v}{ }^{0}}{\mu^{0}}\right)
$$

and where:

$$
\sigma_{i j}^{*}(\underline{x})=-\frac{1}{4 \pi} \int_{V}\left(\frac{1}{\left|\underline{x}-\underline{x}^{\prime}\right|}\right)_{, i j} \sigma_{m}\left(\underline{x}^{\prime}\right) d V^{\prime}
$$

with $\sigma_{i j}^{*}(\underline{x}) \rightarrow 0$ when $|\underline{x}| \rightarrow \infty$.

In Eq. 13, $\sigma^{*}$ is a volume term that is difficult to solve using only the integral form described in Eq. 9 or Eq. 11. Therefore, an incremental time differential equation is now proposed to solve $\underset{\sim}{\sigma^{*}}$. Then, $\underset{\sim}{\sigma^{*}}$ is incrementally computed using Eq. 13 as follows:

$$
\dot{\sigma}_{i j}^{*}(\underline{x})=-\frac{1}{4 \pi} \int_{V^{\prime}}\left(\frac{1}{\left|\underline{x}-\underline{x}^{\prime}\right|}\right)_{, i j} \dot{\sigma}_{m}\left(\underline{x}^{\prime}\right) d V^{\prime}
$$


In Eq. 14, the rate of $\sigma_{m}(\underline{x})$ is obtained using the first constitutive laws in Eq. 1 together with Eq. 9 or Eq. 11, and using Eq. 13 (see [3] for details). Finally, the following time differential equation is obtained for $\sigma^{*}$ :

$$
\begin{aligned}
& \left.\dot{\sigma}_{i j}^{*}(\underline{x})=-\frac{\alpha^{c^{0}}}{4 \pi} \int_{V}\left|\underline{x}-\underline{x}^{\prime}\right|_{, i j k l} R_{k l}\left(\underline{x}^{\prime}\right)\right) d V^{\prime} \\
& +\frac{3 \alpha^{c^{0}}}{2 \pi} \int_{V}\left(\frac{1}{\left|\underline{x}-\underline{x}^{\prime}\right|}\right)_{, i j} R_{m}\left(\underline{x^{\prime}}\right) d V^{\prime}-\frac{\alpha^{c^{0}}}{\alpha^{b^{0}}} \sigma_{i j}^{*}(\underline{x})
\end{aligned}
$$

where:

$$
\begin{aligned}
\alpha^{c^{0}} & =\frac{3 k^{0} \mu^{0}}{3 k^{0}+4 \mu^{0}} \\
\alpha^{b^{0}} & =\frac{3{k_{v}}^{0} \mu_{v}{ }^{0}}{3{k_{v}}^{0}+4 \mu_{v}{ }^{0}}
\end{aligned}
$$

Eq. 15 together with Eq. 9 or Eq. 11 represent the two exact equations of the present incremental internal variables approach that are to be solved simultaneaously in a given time step. In the following, the Eshelby inclusion problem is solved in the context of arbitrary anisotropic ellipsoidal Maxwellian inclusion embedded in an infinite isotropic Maxwellian matrix.

\section{Viscoelastic ellipsoidal Eshelby inclusion}

\subsection{Strain rate concentration equations}

Here, we consider the whole volume $V$ constituted of an ellipsoidal inclusion $I$ with volume $V_{I}$ embedded in an infinite matrix (here considered as the homogeneous reference medium) subjected to $\underset{\sim}{\dot{\varepsilon}^{0}}$ at remote boundaries. The inclusion and the matrix follow a Maxwellian behavior with uniform respective linear elastic $c^{I}$, $\underset{\approx}{c^{0}}$ and inelastic $\underset{\approx}{b^{I}}, \underset{\approx}{b^{0}}$ moduli (and conversely uniform respective linear elastic $\underset{\approx}{s^{I}}, \underset{\approx}{s}$ and inelastic $\underset{\approx}{m^{I}}$, $m_{\approx}^{0}$ compliances). In the following, only the infinite homogeneous reference medium (0) is restricted to be isotropic. Thus, the spatial fluctuations of elastic and linear inelastic properties are given by $\underset{\approx}{\delta}=\underset{\approx}{c^{I}}-\underset{\approx}{c^{0}}$, $\underset{\approx}{\delta s^{I}}=\underset{\approx}{s}-\underset{\approx}{s^{0}}, \underset{\approx}{\delta b^{I}}=\underset{\approx}{b^{I}}-\underset{\approx}{b^{0}}, \underset{\approx}{\delta} m^{I}=\underset{\approx}{m^{I}}-{\underset{\approx}{m}}^{0}$

By denoting $\underset{\sim}{\dot{\varepsilon}^{I}}$ the spatial average of $\dot{\varepsilon}$ over the ellipsoidal inclusion $V_{I}, \dot{\sim}^{I}$ is obtained from Eq. 9:

$$
\underset{\sim}{\dot{\varepsilon}^{I}}=\underset{\sim}{\dot{\varepsilon}^{0}}+\underset{\approx}{T^{c^{I}}}: \underset{\sim}{c^{0}}:{\underset{\sim}{I}}^{I}+\beta \underset{\sim}{\sigma^{* I}}
$$

or from Eq. 11:

$$
\underset{\sim}{\dot{\varepsilon}^{I}}=\underset{\sim}{\dot{\varepsilon}^{0}}+\underset{\approx}{T^{b^{I}}}: \underset{\sim}{b^{0}}: R_{\sim}^{I}+\underset{\sim}{\dot{\sigma}^{* I}}
$$

where for any $\underline{x} \in V_{I}$, the fourth order tensors $\underset{\approx}{T^{0^{I}}}$ and $\underset{\approx}{T^{b^{0^{I}}}}$ defined as:

$$
\begin{aligned}
& \underset{\approx}{T^{c^{0^{I}}}}=\int_{V_{I}} \Gamma_{\approx}^{c^{0}}\left(\underline{x}-\underline{x}^{\prime}\right) d V^{\prime} \\
& \underset{\approx}{T^{b^{0^{I}}}}=\int_{V_{I}} \Gamma^{b^{0}}\left(\underline{x}-\underline{x}^{\prime}\right) d V^{\prime}
\end{aligned}
$$


are uniform tensors due to the Eshelby's property for ellipsoidal inclusions [11]. In Eqs. 18 and 19, $\sigma_{\sim}^{* I}$ is the spatial average of $\sigma^{*}$ over $V_{I}$ and $R_{\sim}^{I}$ is given by:

$$
R_{\sim}^{I}=\underset{\sim}{\delta s}: \dot{\sim}^{I}+\underset{\approx}{\delta m} m^{I}: \sigma^{I}
$$

where $\sigma^{I}$ is the spatial average of $\sigma$ over $V_{I}$.

Using Eq. 21 together with the constituve laws in Eq. 1, Eq. 18 can be rewritten as:

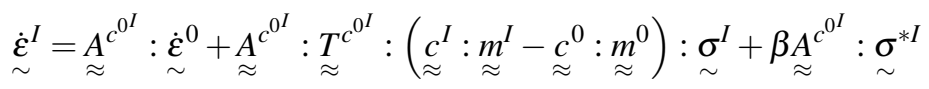

and Eq. 19 can be rewritten as:

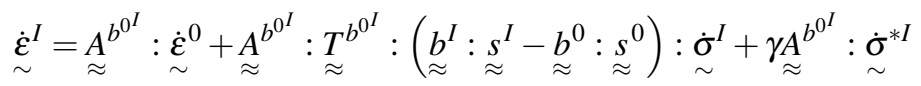

The strain rate concentration tensors $\underset{\approx}{A^{c^{0^{I}}}}$ and $\underset{\approx}{A^{b^{I}}}$ are given by:

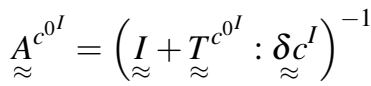

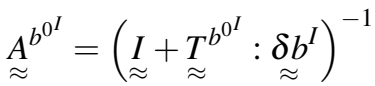

Using Eq. $15, \sigma^{* I}$ is the solution of the following time differential equation:

$$
\dot{\sigma}_{i j}^{* I}=-\frac{\alpha^{c^{0}}}{4 \pi} \psi_{, i j k l}^{I} R_{k l}^{I}+\frac{3 \alpha^{c^{0}}}{2 \pi} \phi_{, i j}^{I} R_{m}{ }^{I}-\frac{\alpha^{c^{0}}}{\alpha^{b^{0}}} \sigma_{i j}^{* I}
$$

In Eq. 25, $\phi^{I}$ and $\psi^{I}$ are harmonic and bi-harmonic potential functions [12, 10, 11] related to the ellipsoidal inclusion $V_{I} . \phi_{, i j}^{I}$ and $\psi_{, i j k l}^{I}$ are given by equation 11.35 (p. 85) in [35]:

$$
\phi_{, i j}^{I}=\int_{V_{I}}\left(\frac{1}{\left|\underline{x}-\underline{x}^{\prime}\right|}\right)_{, i j} d V^{\prime} \quad \psi_{, i j k l}^{I}=\int_{V_{I}}\left|\underline{x}-\underline{x}^{\prime}\right|_{, i j k l} d V^{\prime}
$$

which are uniform when $\underline{x} \in V_{I}$. The expressions of $\phi_{, i j}^{I}$ and $\psi_{, i j k l}^{I}$ for a general ellipsoidal inclusion are given in classic books in micromechanics [35]. $\phi_{, i j}^{I}$ and $\psi_{, i j k l}^{I}$ are contained in $\underset{\approx}{T^{0^{I}}}$, resp. $\underset{\approx}{T^{0^{I}}}$, as follows:

$$
\begin{aligned}
T_{i j k l}^{0^{0^{I}}} & =-\frac{1}{8 \pi \mu^{0}}\left(\phi_{, j l}^{I} \delta_{i k}+\phi_{, i l}^{I} \delta_{j k}\right)+\frac{3 k^{0}+\mu^{0}}{8 \pi \mu^{0}\left(3 k^{0}+4 \mu^{0}\right)} \psi_{, i j k l}^{I} \\
T_{i j k l}^{b^{0^{I}}} & =-\frac{1}{8 \pi \mu_{v}{ }^{0}}\left(\phi_{, j l}^{I} \delta_{i k}+\phi_{, i l}^{I} \delta_{j k}\right)+\frac{3 k_{v}{ }^{0}+\mu_{v}{ }^{0}}{8 \pi \mu_{v}{ }^{0}\left(3 k_{v}{ }^{0}+4 \mu_{v}{ }^{0}\right)} \psi_{, i j k l}^{I}
\end{aligned}
$$

Furthermore, the elastic (resp. inelastic) Eshelby tensors $\underset{\approx}{S^{c^{0}}}$ (resp. $\underset{\approx}{b^{b^{0}}}$ ), for elastic (resp. inelastic) reference

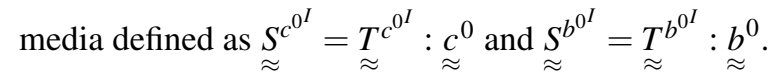

\subsection{Interaction laws}

The interaction law for an arbitrary anisotropic and ellipsoidal inclusion $V_{I}$ embedded in an infinite matrix can be obtained from Eq. 18 or from Eq. 19 and by using the constitutive law in Eq. 1. Using Eq. 1, and after algebraic manipulations, $R^{I}$ defined in Eq. 21 can also be rewritten as follows: 


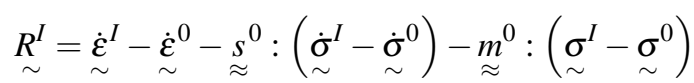

Thus, using Eq. 18 together with Eq. 29 leads to a first form of the interaction law for the linear viscoealstic Eshelby problem:

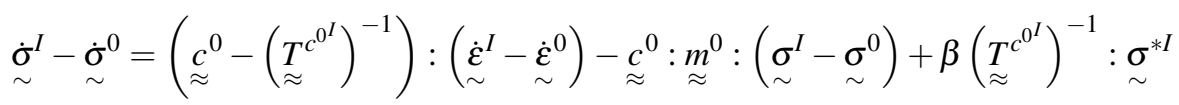

where $\sigma^{0}$ is the uniform remote stress at the boundaries. Using Eq. 19 together with Eq. 29, a second form of the interaction law can be written for the linear viscoealstic Eshelby problem as follows:

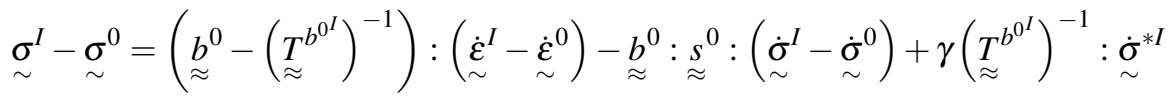

Eqs. 30 and 31 are two forms of the interaction law that are written for the same Maxwellian linear viscoelastic inclusion problem where all the averaged terms are exactly defined. In both equations, the third terms in the second members are new in comparison with other previous approximations starting from the same constitutive equations as Eq. 1 and using the Green's function technique as in $[14,33,36,32,30,31,2$, 27]. These terms describe the complex spatio-temporal integral nature of elastic-inelastic accommodations within the heterogeneous viscoelastic material. Here, they are fully defined through isotropic elastic and inelastic constants of the matrix and the evolution of $\sigma^{* I}$. Note that only the viscoelastic properties of the infinite matrix are isotropic, not the ones related to the inclusion. In [3], the exactness of the interaction law was shown for an arbitrary anisotropic and ellipsoidal inclusion $V_{I}$ embedded in an infinite matrix with isotropic properties in comparison with the exact hereditary approach obtained with the Riemann-Stieltjes integral-based technique [22].

\section{Homogenization and results for two-phase composite materials}

\subsection{Homogenization for two-phase composite materials}

In the case of two-phase composite materials, the Representative Volume Element (RVE) is constituted of several inclusions (phase $I$ ) with volume fraction $f$ embedded in a matrix phase $M$ with volume fraction $(1-f)$. The RVE is subjected at its boundary $\partial V$ to the homogeneous macroscopic strain rate here denoted $\dot{E}$ satisfying:

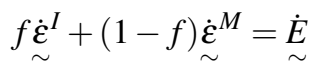

The Mori-Tanaka scheme [34, 49, 1] is based on choosing the infinite homogeneous reference medium 0 as the matrix phase $M$ (i.e. $0=M$ ). This popular scheme is very well suited to estimate the effective properties of two-phase composites with an isotropic distribution of phases, provided the volume fraction $f$ of inclusions is not too large (generally not larger than 0.2). Based on Eq. 22, the strain rate in the phase $I$ is given by:

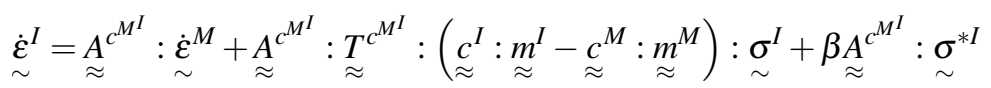

where from Eq. $25, \sigma^{* I}$ is the solution of the following time differential equation:

$$
\dot{\sigma}_{i j}^{* I}=-\frac{\alpha^{c^{M}}}{4 \pi} \psi_{, i j k l}^{I} R_{k l}^{I}+\frac{3 \alpha^{c^{M}}}{2 \pi} \phi_{, i j}^{I} R_{m}^{I}-\frac{\alpha^{c^{M}}}{\alpha^{b^{M}}} \sigma_{i j}^{* I}
$$

and from Eq. 24:

$$
\underset{\approx}{A^{c^{I}}}=\left(\underset{\approx}{I}+\underset{\approx}{c^{M^{I}}}: \underset{\approx}{\delta} c^{I}\right)^{-1}
$$

with $\underset{\approx}{\delta} c^{I}=\underset{\approx}{c^{I}}-\underset{\approx}{c^{M}}$. Using Eq. 33 together with Eq. 32 gives the strain rate in the matrix phase $M$ : 


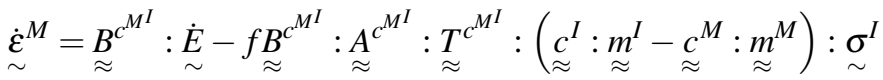

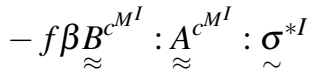

where $\underset{\approx}{B^{c^{I}}}$ is defined as:

$$
\underset{\approx}{B^{M^{I}}}=\left[(1-f) \underset{\approx}{I}+\underset{\approx}{A^{c^{M^{I}}}}\right]^{-1}
$$

In the case of spherical inclusions, $\underset{\approx}{T^{M^{I}}}$ is given by:

$$
\underset{\approx}{T^{M^{I}}}=\frac{1}{3 k^{M}+4 \mu^{M}} \underset{\approx}{\approx}+\frac{3\left(k^{M}+2 \mu^{M}\right)}{5 \mu^{M}\left(3 k^{M}+4 \mu^{M}\right)} \underset{\approx}{K}
$$

and the expressions of $\phi_{, i j}^{I}$ and $\psi_{, i j k l}^{I}$ (Eq. 26) are simplified to the following expressions [46, 35]:

$$
\phi_{, i j}^{I}=-\frac{4 \pi}{3} \delta_{i j} \quad \psi_{, i j k l}^{I}=-\frac{8 \pi}{15}\left(\delta_{i j} \delta_{k l}+\delta_{i k} \delta_{j l}+\delta_{i l} \delta_{j k}\right)
$$

Therefore, the explicit forms of $\underset{\approx}{A^{M^{I}}}$ and $\underset{\approx}{B^{M^{I}}}$ follow from Eq. 38 together with Eq. 35 and Eq. 37 , respectively. Furthermore, Eq. 34 for spherical inclusions simplifies to:

$$
\dot{\sigma}_{i j}^{* I}=\frac{4}{15} \alpha^{c^{M}}\left(R_{i j}{ }^{I}-6 R_{m}{ }^{I} \delta_{i j}\right)-\frac{\alpha^{c^{M}}}{\alpha^{b^{M}}} \sigma_{i j}^{* I}
$$

Therefore, the average stress rates in each phase $\dot{\sigma}^{I}$ and $\dot{\sigma}^{M}$ are obtained from Eqs. 33, 36, and from the constitutive laws (Eq. 1). Lastly, the overall stress $\underset{\sim}{\widetilde{\Sigma}}$ of the composite is incrementally obtained from the stress rate averaging rule:

$$
\underset{\sim}{\dot{\Sigma}}=f \underset{\sim}{\dot{\sigma}^{I}}+(1-f) \underset{\sim}{\dot{\sigma}^{M}}
$$

In the following applications and for simplicity, both phases (inclusions and matrix) will have isotropic properties and the inclusions will be supposed to be spherical.

\subsection{Results and discussion}

\subsubsection{Comparison with a hereditary approach}

A first type of application is to show that the present time-incremental Eshelby-based homogenization scheme is able to easily retrieve the same results as the ones obtained from hereditary approaches using the correspondence principle and inverse Laplace or Laplace-Carson transforms. One of the main shortcoming of these approaches compared to the present approach derived in the time domain is that simple analytical solutions remains limited to particular cases even for a composite constituted of two phases with a Mawellian behavior and isotropic properties. As an example, let us consider the case developed by [24] who used a Mori-Tanaka scheme for two-phase composites under constant strain rate loading in the case where the spherical inclusions with volume fraction $f=0.2$ are elastic and the inelastic (viscous) behavior of the matrix phase is supposed to be pressure independent. Starting from the effective bulk and shear moduli in the transformed Laplace domain, the equations 5.5 and 5.6 in [24] were obtained by Laplace inversion. By considering Eqs. 32 to 41 of the present model, the exact solutions for purely hydrostatic and purely deviatoric loadings are easily retrieved. As an illustration for the application of the present exact time-incremental approach to this specific case, the materials parameters used for this comparison are reported in Table 1. Fig. 1(a) shows the evolution of the overall hydrostatic stress $\Sigma_{k k}$ as a function of the overall dilatational strain $E_{k k}$ whereas Fig. 1(b) displays the evolution of the overall (deviatoric) shear stress $\Sigma_{12}$ as a function of the overall shear strain $E_{k k}$, for both applied $\dot{E}_{k k}$ and $\dot{E}_{12}$ equal to $10^{-4} s^{-1}$. The different asymptotic states are large times were also checked to be consistent with the expressions given by 
[24] (see their equations 5.7 and 5.8). For large times or large strains, the shear stress tends to a saturation stress whereas the dilatational stress response is characterized by a constant tangent modulus equal to the effective elastic bulk modulus of the composite.

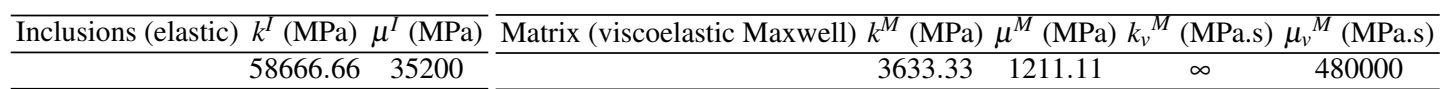

Table 1 Materials parameters for the introduced two-phase composite with elastic inclusions and Maxwellian matrix for comparison with the hereditary formulation of [24]. For the simulations, the volume fraction of inclusions is set to $f=0.2$.
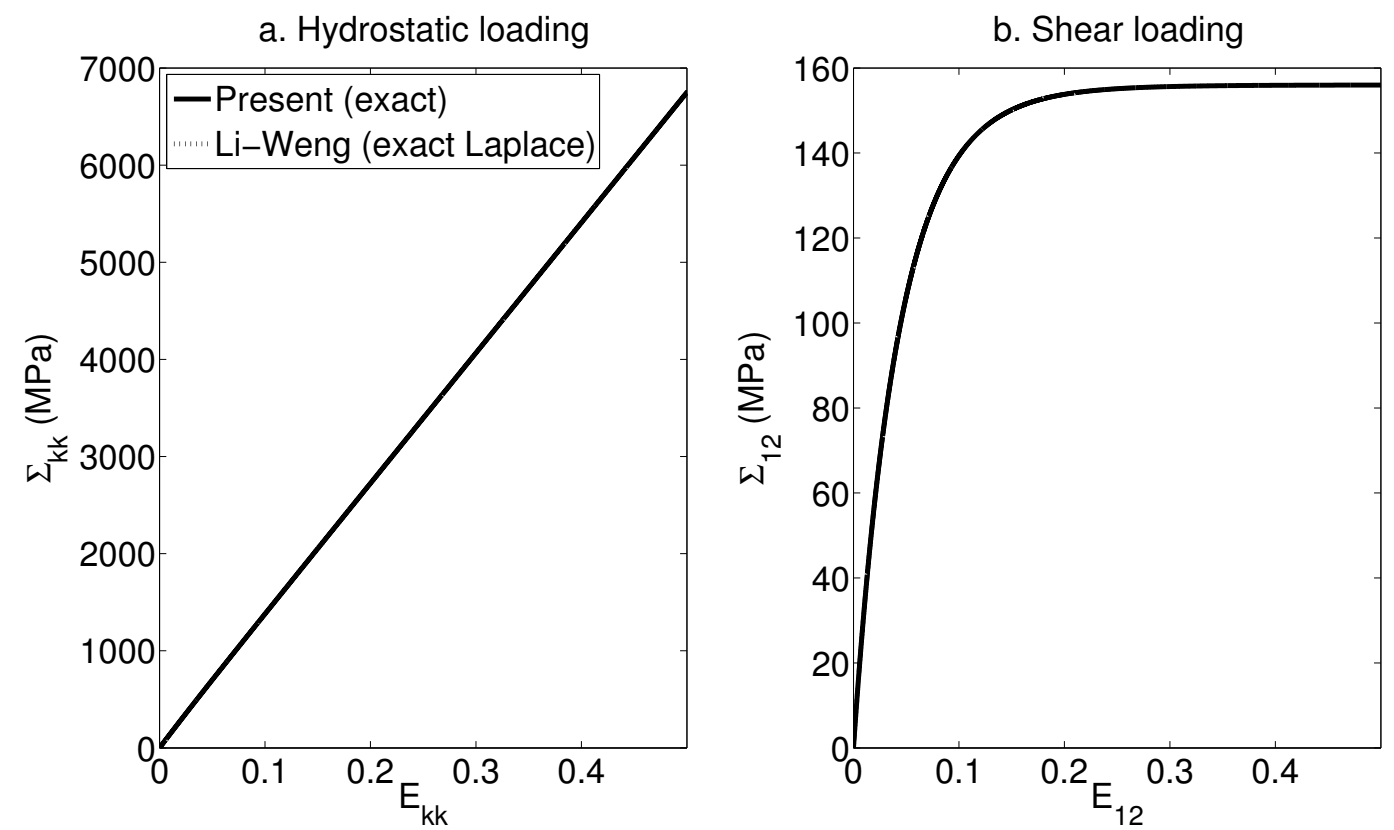

Fig. 1 Overall stress-strain responses of a two-phase composite with spherical elastic inclusions of volume fraction $f=0.2$ and with a matrix phase exhibiting a Maxwell behavior such that the bulk viscoelastic modulus is restricted to the bulk elastic modulus (see Table 1). (a) Overall hydrostatic stress $\Sigma_{k k}$ as a function of $E_{k k}$ for an applied dilatational strain rate $\dot{E}_{k k}=$ $10^{-4} s^{-1}$, (b) Overall shear stress $\Sigma_{12}$ as a function of $E_{12}$ for an applied shear strain rate $\dot{E}_{12}=10^{-4} s^{-1}$. The present model exactly matches the [24]'s analytical solutions which are superimposed to the present's model responses. 


\subsubsection{Comparison with another exact internal variable approach}

In the case of linear Maxwellian compressible two-phase composites with homogeneous elastic properties, exact solutions were recently using a Mori-Tanaka homogenization scheme by [39]. Their approach is based on an internal variable formulation inspired from the collocation method. The evolution laws of the average stresses in both phases were obtained with inverse Laplace-Carson transforms in [39], knowing the overall behavior from the internal variables evolutions. For comparison, in the present method, the average phase behaviors and the effective behavior are directly obtained from Eqs. 32 to 41 in the time domain without the need of Laplace-Carson inversions. The inclusions are assumed spherical with a volume fraction of $f=0.2$. In order to assess the present model in comparison with the [39]'s solutions, four cases are considered as reported in Table 2. The common parameters in all cases are the elastic shear moduli $\mu^{I}=\mu^{M}=50000 \mathrm{MPa}$ and the inelastic shear modulus of the matrix $\mu_{v}{ }^{M}=10000 \mathrm{MPa}$.s. Both phases have same elastic properties like in [39]. The inclusions are also considered with mechanical contrasts 100 (cases 1 and 2), resp 1/100 (cases 3 and 4), with respect to the matrix on the bulk/shear elastic/inelastic moduli. In the "case 1 " and the "case 3", the phase behaviors are assumed compressible elastic (uniform elastic Poisson ratio: $v_{e}=0.05$ ) and incompressible inelastic (uniform inelastic Poisson ratio $v_{v} \sim 0.5$ ). In the "case 2 " and the "case 4", a full compressible viscoelasticity in both phases is assumed with constants $v_{e}=0.05$ and $v_{v}=0.3$.

\begin{tabular}{ccccc}
\hline Case & $k^{I}=k^{M}(\mathrm{MPa})$ & $k_{v}{ }^{M}$ (MPa.s) & $k_{v}{ }^{I}$ (MPa.s) & $\mu_{v}{ }^{I}$ (MPa.s) \\
\hline Case 1 & 38890 & $\infty$ & $\infty$ & 1000000 \\
\hline Case 2 & 38890 & 21666.66 & 2166666 & 1000000 \\
\hline Case 3 & 38890 & $\infty$ & $\infty$ & 100 \\
\hline Case 4 & 38890 & 21666.66 & 216.6666 & 100 \\
\hline
\end{tabular}

Table 2 Materials parameters introduced for the first illustrations (comparisons with [39]'s approach): compressible elasticity/incompressible inelasticity (cases 1 and 3), compressible elasticity/inelasticity (cases 2 and 4).

A uniaxial tension-compression test is simulated with the following prescribed macroscopic strain rate $\underset{\sim}{\dot{E}}=\dot{E}_{11}\left(\begin{array}{ccc}1 & 0 & 0 \\ 0 & -0.5 & 0 \\ 0 & 0 & -0.5\end{array}\right)$ with $\dot{E}_{11}=10^{-4} s^{-1}$ for tension and $\dot{E}_{11}=-10^{-4} s^{-1}$ for compression. For comparisons of the overall responses given by the present internal variables approach with the [39] solutions, equations 10, 18 and Table 1 in [39] are used.

Fig. 2 shows the overall stress $\Sigma_{11}$ cyclic responses up to $t=20 \mathrm{~s}$ for cases 1 and 2 (high mechanical contrast of 100 with the matrix) as functions of $E_{11}$ (Fig. 2(a)) and physical time $t$ (Fig. 2(b)). It was checked that both present and [39] models give the same responses (the curves for both models are superimposed) regarding the transient regimes between overall elastic and inelastic states and the asymptotic state during tension and compression. In particular, the differences between compressible elasticity only and fully compressible viscoelasticity are well captured by the present model, especially when the loading changes from tension to compression.

For cases 3 and 4 (high mechanical contrast of 1/100 with respect to the matrix), Fig. 3 verifies that both present and [39] models also give the same responses up to $t=10 \mathrm{~s}$ (the curves for both models are superimposed) regarding the transient regimes between overall elastic and inelastic states and the (inelastic) asymptotic states during tension and compression. As a function of materials properties, the composite responses have different transient and asymptotic states which are observable on Fig. 2 and Fig. 3. The effect of elastic or viscoelastic compressibility is observed in the same way with both models. The present formulation also well captures the stress history due to materials properties in the transient effective behavior during the reverse compression loading that are more important than during the first tensile stage.

In order to refine the analysis, the time evolutions of the uniaxial phase average stresses $\sigma_{11}^{I}$ and $\sigma_{11}^{M}$ corresponding to cases 1 to 4 are reported in Figs. 4 and 5 to see the differences due to two different mechanical constrasts on the phase responses, i.e. 100 in Figs. 4(a) and 5(a), and 1/100 in Figs. 4(b) and 5 (b). 
a.

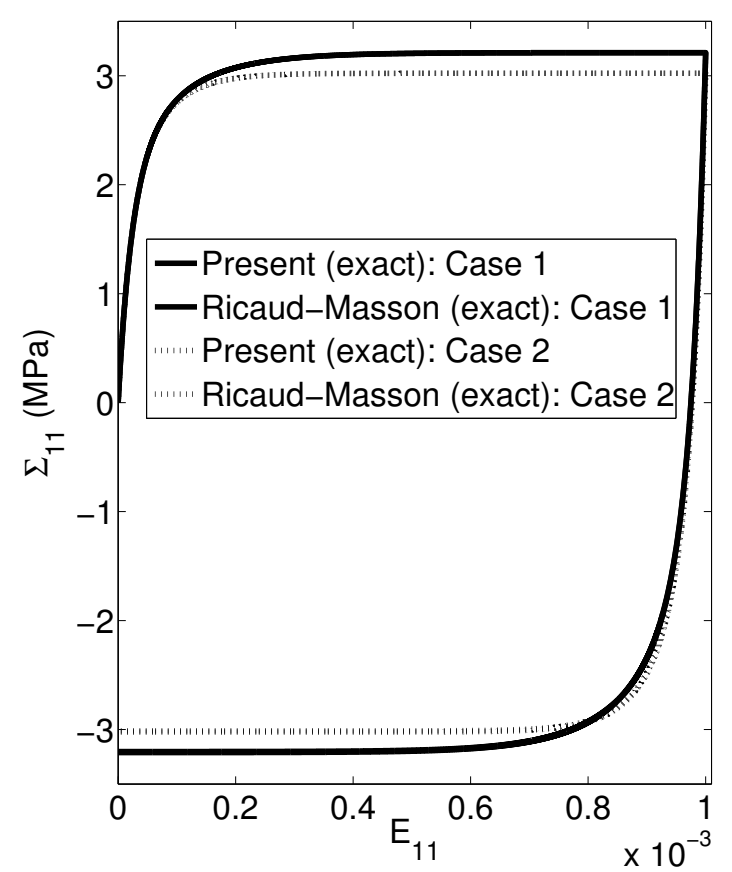

b.

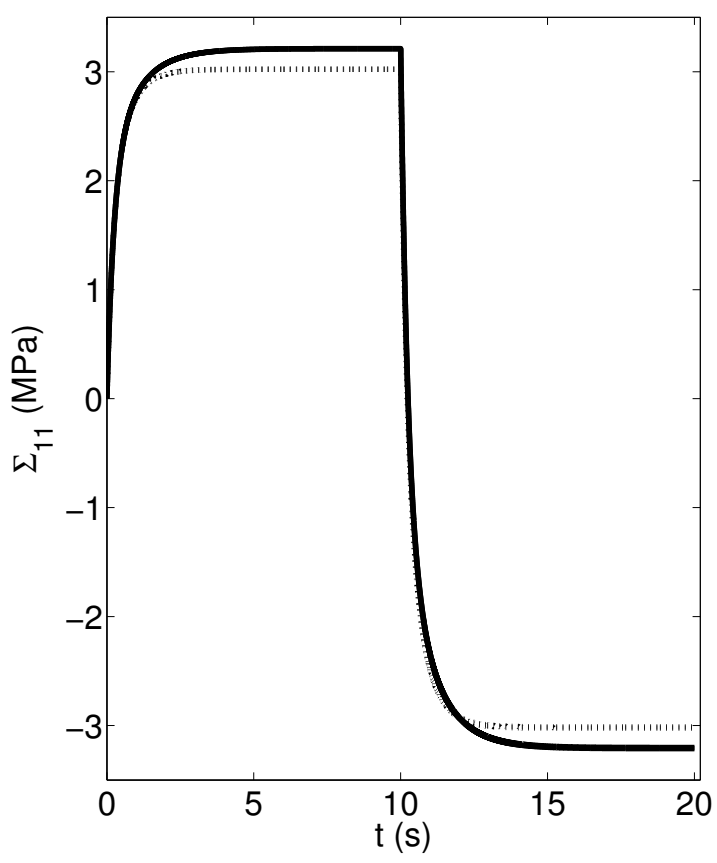

Fig. 2 Cases 1 and 2 (see Table 2 for materials parameters): overall tension-compression uniaxial stress $\Sigma_{11}$ responses predicted by the present linear homogenization Mori-Tanaka approach based on the exact interaction law for the Eshelby's inclusion problem (Eqs. 32 to 41) or by the [39]'s Mori-Tanaka approach, as functions of applied macroscopic strain $E_{11}$ (a) and physical time $t$ (b). The present model matches exactly the [39]'s solutions which are superimposed to the present's model responses.

a.

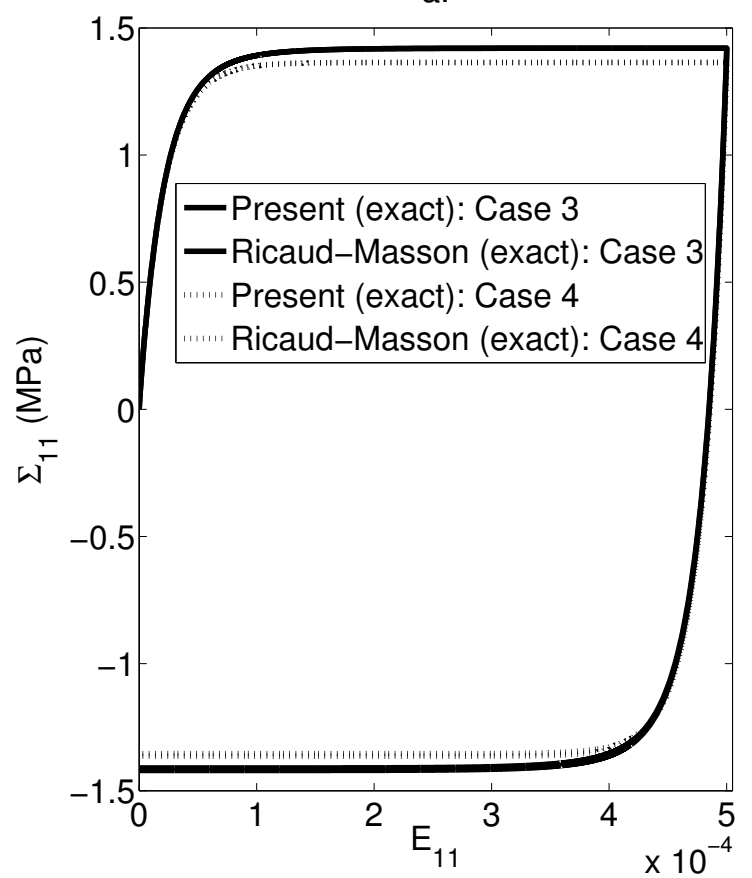

b.

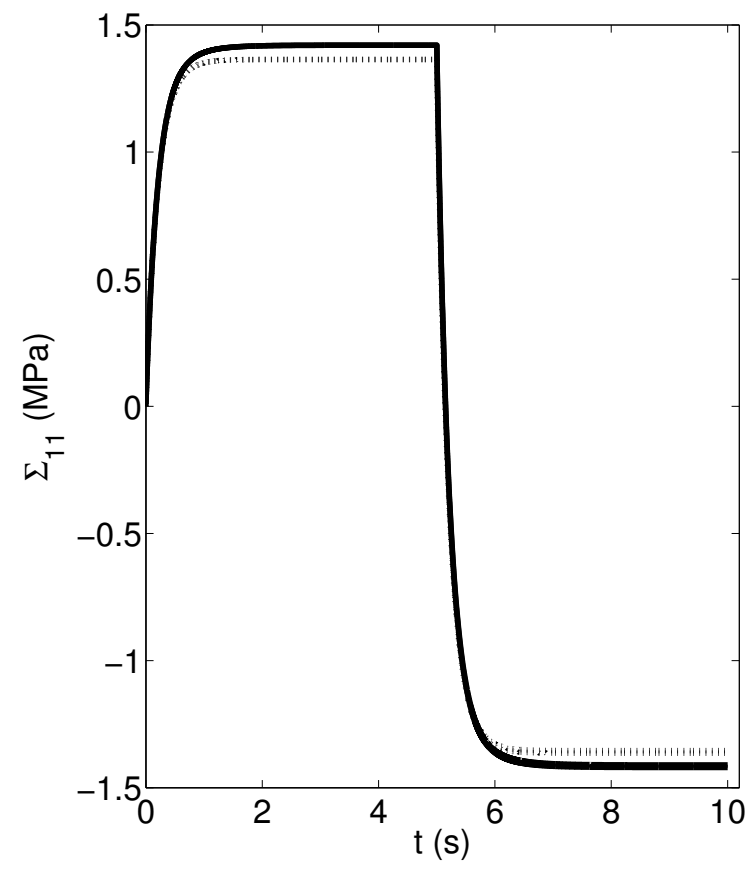

Fig. 3 Cases 3 and 4 (see Table 2 for materials parameters): overall tension-compression uniaxial stress $\Sigma_{11}$ responses predicted by the present linear homogenization Mori-Tanaka approach based on the exact interaction law for the Eshelby's inclusion problem (Eqs. 32 to 41) or by the [39]'s Mori-Tanaka approach, as functions of applied macroscopic strain $E_{11}$ (a) and physical time $t$ (b). The present model matches exactly the [39]'s solutions which are superimposed to the present's model responses. 
a. Case 1

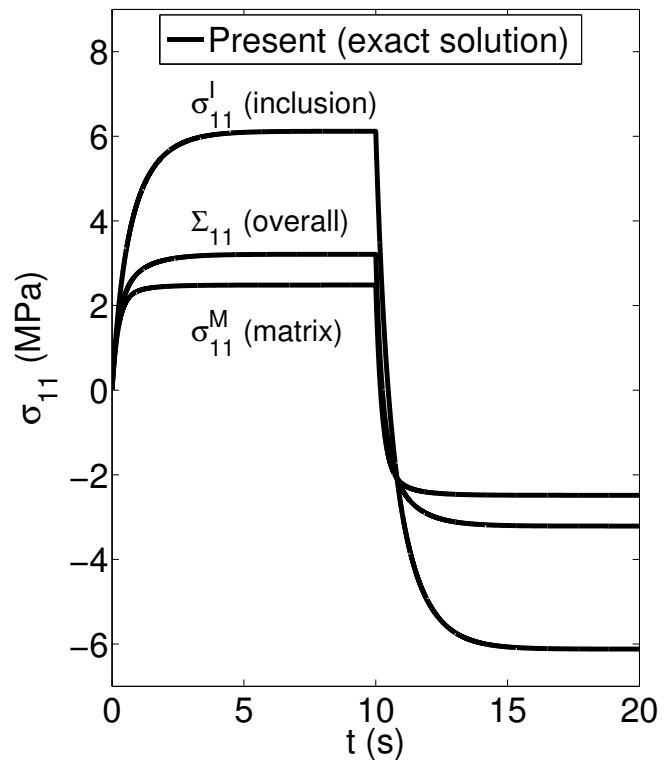

b. Case 3

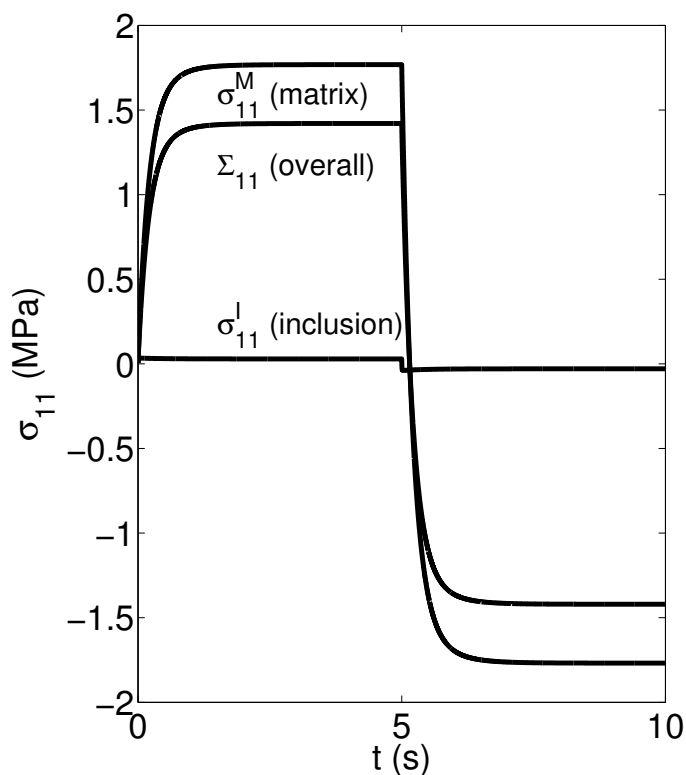

Fig. 4 Tension-compression simulations in the case 1 (a) and in the case 3 (b) (see Table 2 for materials parameters): time evolution of uniaxial overall stress $\Sigma_{11}$ and phase average stress $\sigma_{11}$ responses predicted by the homogenization Mori-Tanaka approach based on the exact interaction law (Eqs. 32 to 41 ).

a. Case 2

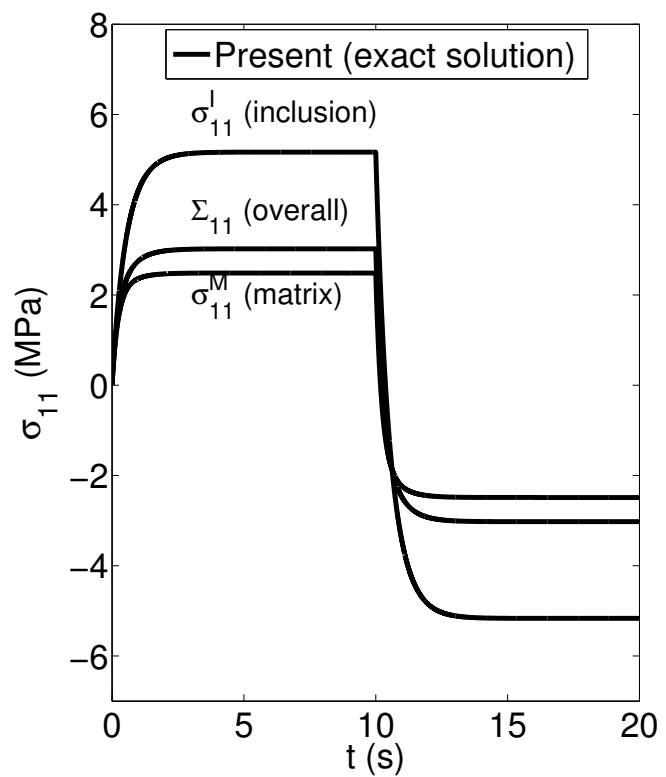

b. Case 4

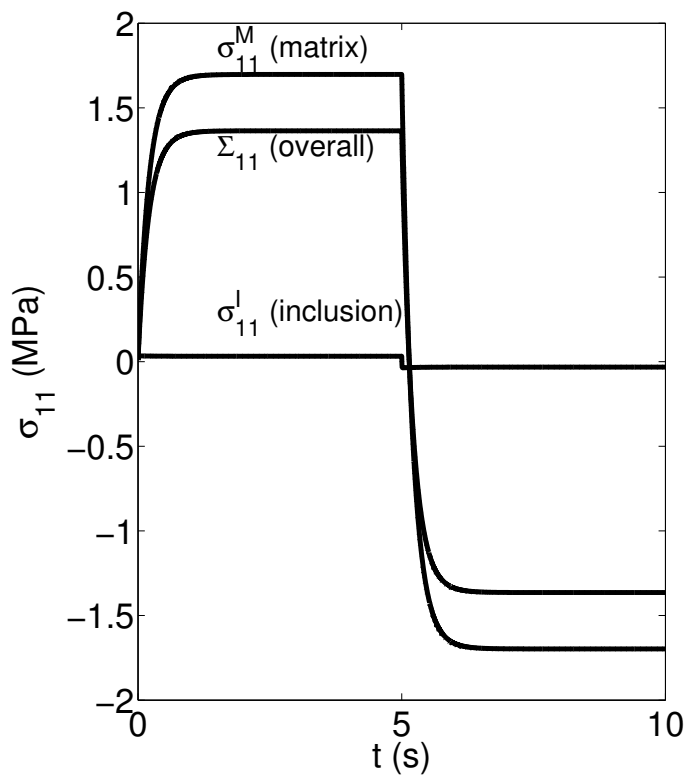

Fig. 5 Tension-compression simulations in the case 2 (a) and in the case 4 (b) (see Table 2 for materials parameters): time evolution of uniaxial overall stress $\Sigma_{11}$ and phase average stress $\sigma_{11}$ responses predicted by the homogenization Mori-Tanaka approach based on the exact interaction law (Eqs. 32 to 41). 


\subsubsection{Comparisons with the "translated fields" method and the "additive law"}

For comparisons with previous internal variable models containing approximations like the "translated fields" method [36, 31, 2] and the "additive interaction" law [14, 33, 32], a third application of the present time-incremental Eshelby-based model is now focused on heterogeneous and compressible elasticity in addition to heterogeneous incompressible inelasticity for inclusions stiffer than the matrix (case A) or the matrix stiffer than the inclusions (case B). Interestingly, such a case was recently treated by [15] with different approximate models (see their Fig. 4) and using inverse Laplace-Carson to find the exact time evolution of the strain rate in the spherical inclusion. This application can be performed assuming a diluted concentration of Eshelby inclusions, i.e. in the limiting case where $f \rightarrow 0$ using the present Mori-Tanaka homogenization scheme. The common materials properties describe elastic compressible properties for the matrix and inclusions with same elastic Poisson ratios $v_{e}^{I}=v_{e}{ }^{M}=0.05$, an elastic Young's modulus for the matrix of $E^{M}=200 \mathrm{MPa}$, and, an incompressible inelasticity for the matrix given by $\mu_{v}{ }^{M}=1 / 3 \mathrm{MPa}$.s. The other parameters are introduced to account for different mechanical contrasts. More specifically, two different elastic Young's moduli and inelastic shear moduli ratios are considered in Table 3.

\begin{tabular}{ccc}
\hline Case & $E^{I} / E^{M}$ & $\mu_{v}{ }^{1} / \mu_{v}{ }^{M}$ \\
\hline Case A: inclusions stiffer than matrix & 3 & 10 \\
\hline Case B: matrix stiffer than inclusions & $1 / 3$ & $1 / 10$ \\
\hline
\end{tabular}

Table 3 Materials parameters introduced for the third application for comparisons with approximate models.

Following [15], a uniaxial tension-compression test is simulated with the following applied strain rate $\underset{\sim}{\dot{E}}=\dot{E}_{11}\left(\begin{array}{ccc}1 & 0 & 0 \\ 0 & -0.5 & 0 \\ 0 & 0 & -0.5\end{array}\right)$ with $\dot{E}_{11}=1 s^{-1}$ for tension and $\dot{E}_{11}=-1 s^{-1}$ for compression. Fig. 6 reports the axial strain rate $\dot{\varepsilon}_{11}^{I}$ evolution in the inclusion along the tension-compression axis as a function of time $t$ obtained with the present model for "case A" (Fig. 5(a)) and for "case B" (Fig. 6(b)) according to the parameters given in Table 3. By comparing such evolutions with the exact solutions obtained by the correspondence principle and Laplace-Carson inversion, both approaches give the same results. In particular, for "case B", Fig. 6(b) displays a peak strain rate at the onset of the tensile and compression stages with the present exact solution which is not really captured by the "translated fields" method and by the "additive interaction" law (see Fig. 6(b)). As reported in [15], the "translated fields" method and the "additive interaction" law were the best approximate models for this application. This application demonstrates the advantage of the present model to obtain the exact strain rate evolution in the inclusion without the need of Laplace-Carson inversion. The present approach also improves the predictions of previous approximate models that were seen to be unable to match the exact solutions. 

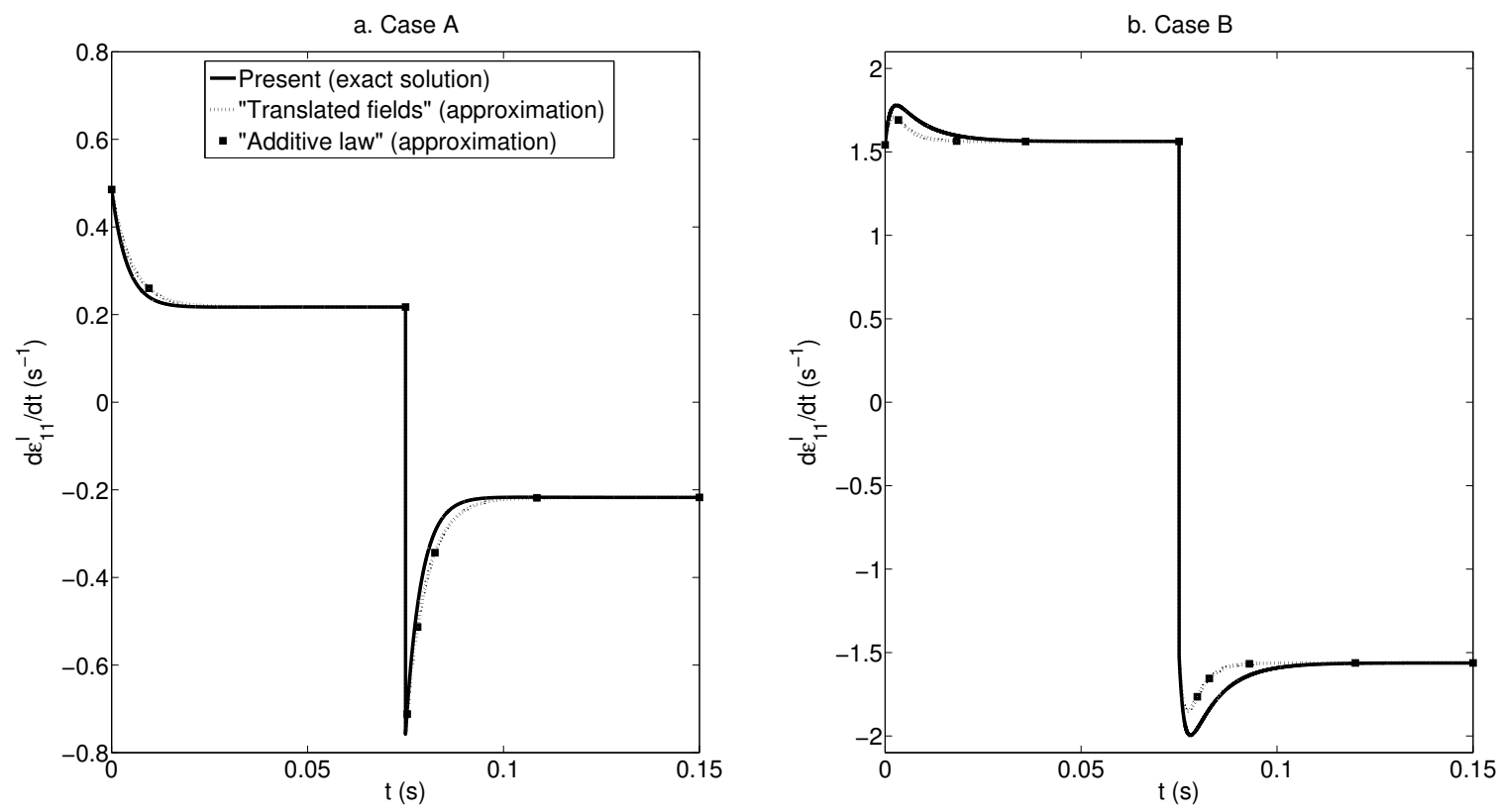

Fig. 6 Time evolution of axial strain rate $\dot{\varepsilon}_{11}^{I}$ in the inclusion along the tension-compression axis predicted by the present linear homogenization Mori-Tanaka approach based on the exact interaction law for the Eshelby's inclusion problem (Eqs. 32 to 41) in the "case A" (a) and ine the "case B" (b). For both cases, the present model (solid lines) matches exactly the inverse Laplace-Carson solutions reported in [15]. For comparisons, the time evolutions of $\dot{\varepsilon}_{11}^{I}$ given by the approximate "translated fields" method (dashed lines) and "additive interaction" law (points) are reported.

\section{Conclusions}

A time-incremental internal variables homogenization scheme for Maxwellian linear viscoelastic heterogeneous materials has been presented. The formulation is based on isotropic elastic and inelastic homogeneous reference media associated with the strain rate integral equation. The complete solution is obtained through the introduction of the variable $\sigma^{*}$, which reflects a volume term in the integral equation of the heterogeneous viscoelastic problem. This variable is exactly solved by a time-differential equation. The major advantage of the present internal variables formulation compared to previous internal variables approaches containing approximations is the derivation of the exact interaction laws for the Eshelby ellipsoidal inclusion problem. In the case of a homogenization Mori-Tanaka scheme for an isotropic distribution of phases, the present internal variables formulation may be considered as another alternative way to fasten some numerical calculations in structures made of linear viscoelastic composites. The present time-incremental internal variables approach can be applied to ageing linear viscoelastic behaviors without any major difficulty in contrast with Riemann-Stieltjes integral-based hereditary homogenization approaches. The extensions to more refined homogenization schemes taking into account ellipsoidal distributions of phases [38, 51] are left for future work. Furthermore, the present framework can be extended to other types of rheological models in linear viscoelasticity, such as Kelvin-Voigt, Burgers and Generalized Maxwell models.

\section{References}

1. Benveniste, Y.: A new approach to the application of Mori-Tanaka's theory in composite materials. Mech. Mater. 6(2), 147-157 (1987)

2. Berbenni, S., Capolungo, L.: A MoriTanaka homogenization scheme for non-linear elasto-viscoplastic heterogeneous materials based on translated fields: An affine extension. C. R. Mecanique 343, 95-106 (2015)

3. Berbenni, S., Dinzart, F., Sabar, H.: A new internal variables homogenization scheme for linear viscoelastic materials based on an exact Eshelby interaction law. Mech. Mater. 81, 110-124 (2015) 
4. Brassard, L., Stainier, L., Doghri, I., Delannay, L.: Homogenization of elasto-(visco)plastic composites based on an incremental variational principle. Int. J. Plast. 36, 86-112 (2012)

5. Brenner, R., Masson, R., Castelnau, O., Zaoui, A.: A "quasi-elastic" affine formulation for the homogenised behaviour of nonlinear viscoelastic polycrystals and composites. Eur. J. Mech. A. Solids 21, 943-960 (2002)

6. Brinson, L.C., Lin, W.S.: Comparison of micromechanics methods for effective properties of multiphase viscoelastic composites. Compos. Struct. 41, 353-367 (1998)

7. Christensen, R.M.: Viscoelastic properties of heterogeneous media. J. Mech. Phys. Solids 17, 23-41 (1969)

8. Coulibaly, M., Sabar, H.: Micromechanical modeling of linear viscoelastic behavior of heterogeneous materials. Arch. Appl. Mech. 81, 345-359 (2011)

9. DeBotton, G., Tevet-Deree, L.: The response of a fiber-reinforced composite with a viscoelastic matrix phase. J. Compos. Mater. 38, 1255-1277 (2004)

10. Dyson, F.: The potentials of ellipsoids of variable densities. Q. J. Pure Appl. Math. 25, 259-288 (1891)

11. Eshelby, J.D.: The determination of the elastic field of an ellipsoidal inclusion and related problems. Proc. Roy. Soc. London A. 241, 376-396 (1957)

12. Ferrers, N.: On the potentials of ellipsoids, ellipsoidal shells, elliptic laminae and elliptic rings of variable densities. Q. J. Pure Appl. Math. 14, 1-22 (1877)

13. Hashin, Z.: The inelastic inclusion problem. Int. J. Eng. Sci. 7, 11-36 (1969)

14. Kouddane, R., Molinari, A., Canova, G.R.: Self-consistent modelling of heterogeneous viscoelastic and elastoplastic materials. In: Mecamat 91: Large Plastic Deformations, Fundamentals and Application to Metal Forming, pp. 129-141. C. Teodosiu, J-.L. Raphanel, F. Sidoroff (Eds), Balkema,Rotterdam (1993)

15. Kowalczyk-Gajewska, K., Petryk, H.: Sequential linearization method for viscous/elastic heterogeneous materials. Eur. J. Mech. A Solids 30, 650-664 (2011)

16. Kröner, E.: Zur plastischen Verformung des Vielkristalls. Acta Metall. 9, 155 - 161 (1961)

17. Kröner, E.: Modified Green functions in the theory of heterogeneous and/or anisotropic linearly elastic media. In: Micromechanics and Inhomogeneity. The Toshio Mura 65th Anniversary Volume, pp. 197-211. G.J. Weng, M. Taya, H. Abe (eds.), Springer Verlag, New York (1990)

18. Kunin, I.A.: Elastic media with microstructure II: Three-dimensional models. In: Springer Series in Solid-State Sciences 44. E. Kröner (Ed.), Springer Berlin (1983)

19. Lahellec, N., Suquet, P.: Effective behavior of linear viscoelastic composites: A time-integration approach. Int. J. Solids Struct. 44, 507-529 (2007)

20. Lahellec, N., Suquet, P.: On the effective behavior of non linear inelastic composites: I. Incremental variational principles. J. Mech. Phys. Solids 55, 1932-1963 (2007)

21. Lavergne, F., Sab, K., Sanahuja, J., Bornert, M., Toulemonde, C.: Homogenization schemes for aging linear viscoelastic matrix-inclusion composite materials with elongated inclusions. Int. J. Solids Struct. 80, 545-560 (2016)

22. Laws, N., McLaughlin, R.: Self-consistent estimates for the viscoelastic creep compliance of composite materials. Proc. Roy. Soc. London A 359, 251-273 (1978)

23. Levesque, M., Gilchrist, M.D., Bouleau, N., Derrien, K., Baptiste, D.: Numerical inversion of the Laplace-Carson transform applied to homogenization of randomly reinforced linear viscoelastic media. Comput. Mech. 40, 771-789 (2007)

24. Li, J., Weng, G.J.: Strain-rate sensitivity, relaxation behavior and complex moduli of a class of isotropic viscoplastic composites. ASME J. Eng. Mater. Tech. 116, 495-504 (1994)

25. Li, J., Weng, G.J.: A secant-viscosity approach to the time-dependent creep of an elastic-viscoplastic composite. J. Mech. Phys. Solids 45(7), 1069-1083 (1997)

26. Mandel, J.: Cours de Mécanique des Milieux Continus. Gauthiers-Villars, Paris (1966)

27. Mareau, C., Berbenni, S.: An affine formulation for the self-consistent modeling of elasto-viscoplastic heterogeneous materials based on the translated field method. Int. J. Plast. 64, 134-150 (2015)

28. Masson, R., Brenner, R., Castelnau, O.: Incremental homogenization approach for ageing viscoelastic polycrystals. C. R. Mecanique 340, 378-386 (2012)

29. Masson, R., Zaoui, A.: Self-consistent estimates for the rate-dependent elastoplastic behaviour of polycrystalline materials. J. Mech. Phys. Solids 47, 1543-1568 (1999)

30. Mercier, S., Molinari, A.: Homogenization of elasticviscoplastic heterogeneous materials: self-consistent and moritanaka schemes. Int. J. Plast. 25, 1024-1048 (2009)

31. Mercier, S., Molinari, A., Berbenni, S., Berveiller, M.: Comparison of different homogenization approaches for elasticviscoplastic materials. Modell. Simul. Mater. Sci. Eng. 20, 024,004 (2012)

32. Molinari, A.: Averaging models for heterogeneous viscoplastic and elastic-viscoplastic materials. ASME J. Eng. Mater. Tech. 124, 62-70 (2002)

33. Molinari, A., Ahzi, S., Kouddane, R.: On the self-consistent modelling of elastic-plastic behavior of polycrystals. Mech. Mater. 26, 43-62 (1997)

34. Mori, T., Tanaka, K.: Average stress in matrix and average elastic energy of materials with misfitting inclusions. Acta Metall. 21, 571-574 (1973)

35. Mura, T.: Micromechanics of defects in solids. Kluwer Academic Publishers, Dordrecht, The Netherlands (1987)

36. Paquin, A., Sabar, H., Berveiller, M.: Integral formulation and self-consistent modelling of elasto-viscoplastic behavior of heterogeneous materials. Arch. Appl. Mech. 69, 14-35 (1999)

37. Pierard, O., Doghri, I.: An enhanced affine formulation and the corresponding numerical algorithms for the mean-field homogenization of elasto-viscoplastic composites. Int. J. Plast. 22, 131-157 (2006)

38. Ponte Castañeda, P., Willis, J.R.: The effect of spatial distribution on the effective behavior of composite materials and cracked media. J. Mech. Phys. Solids 43, 1919-1951 (1995)

39. Ricaud, J.M., Masson, R.: Effective properties of linear viscoelastic heterogeneous media: internal variables formulation and extension to ageing behaviours. Int. J. Solids Struct. 46, 1599-1606 (2009) 
40. Rougier, Y., Stolz, C., Zaoui, A.: Representation spectrale en viscoelasticite lineaire des materiaux heterogenes. C. R. Acad. Sci. Paris Ser. II 316, 1517-1522 (1993)

41. Rougier, Y., Stolz, C., Zaoui, A.: Self-consistent modelling of elastic-viscoplastic polycrystals. C. R. Acad. Sci. Paris Ser. IIb 318, 145-151 (1994)

42. Sabar, H., Berveiller, M., Favier, V., Berbenni, S.: A new class of micro-macro models for elastic-viscoplastic heterogeneous materials. Int. J. Solids Struct. 39, 3257-3276 (2002)

43. Sanahuja, J.: Effective behavior of aging linear viscoelastic composites: homogenization approach. Int. J. Solids Struct. 50, 2846-2856 (2013)

44. Suquet, P.: Elements of homogenization for inelastic solid mechanics. In: Homogenization techniques for Composite media, pp. 193-278. E. Sanchez-Palencia and A. Zaoui (Eds), Springer Berlin (1987)

45. Turner, P.A., Tomé, C.N.: Self-consistent modeling of visco-elastic polycrystals: Application to irradiation creep and growth. J. Mech. Phys. Solids 41(7), 1191-1211 (1993)

46. Walpole, L.J.: Elastic behavior of composite materials: theoretical foundations. Adv. Appl. Mech. 21, 169-242 (1981)

47. Wang, Y.M., Weng, G.J.: The influence of inclusion shape on the overall viscoelastic behavior of composites. ASME J. Appl. Mech. 59(3), 510-518 (1992)

48. Weng, G.J.: Self-consistent determination of time-dependent behavior of metals. ASME J. Appl. Mech. 48, 41-46 (1981)

49. Weng, G.J.: Some elastic properties of reinforced solids with special reference to isotropic ones containing spherical inclusions. Int. J. Eng. Sci. 22(7), 845-856 (1984)

50. Weng, G.J.: A self-consistent relation for the time-dependent creep of polycrystals. Int. J. Plast. 9, 181-198 (1993)

51. Zheng, Q.S., Du, D.X.: An explicit and universally applicable estimate for the effective properties of multiphase composite which accounts for inclusion distribution. J. Mech. Phys. Solids 49, 2765-2788 (2001) 